\title{
Phénomènes de canalisation. Applications à la caractérisation des échantillons cristallins.
}

\author{
François Abel, \\ GPS, Universités Paris 6 et Paris 7, UMR 7588 du CNRS, \\ Tour 23, 2 place Jussieu, F-75251 PARIS CEDEX 05, FRANCE \\ abel@gps.jussieu.fr
}

\section{PHÉNOMÈNES DE CANALISATION}

Lors de l'irradiation d'échantillons cristallins avec des ions, si le faisceau est aligné avec une rangée ou un plan cristallographique de bas indices, on observe une réduction, d'un facteur de l'ordre de 50 pour un bon cristal, du rendement des interactions à petit paramètre d'impact - diffusion élastique à grand angle, réactions nucléaires, émissions de RX de cœur -.

Ceci est expliqué par le fait que dans le cas de l'alignement, les paramètres d'impact des interactions successives entre les ions incidents et les atomes du cristal sont très fortement corrélés. La trajectoire d'un ion peut alors être considérée comme déterminée par un continuum de potentiel qui est le potentiel d'interaction ion-atome moyenné le long de la direction, ou plan, cristallographique considérée. Elle présente des oscillations dans les «canaux» autour des rangées ou dans l'intervalle entre deux plans. Ce phénomène appelé «canalisation » focalise les ions loin des rangées atomiques interdisant ainsi les interactions à petit paramètre d'impact.

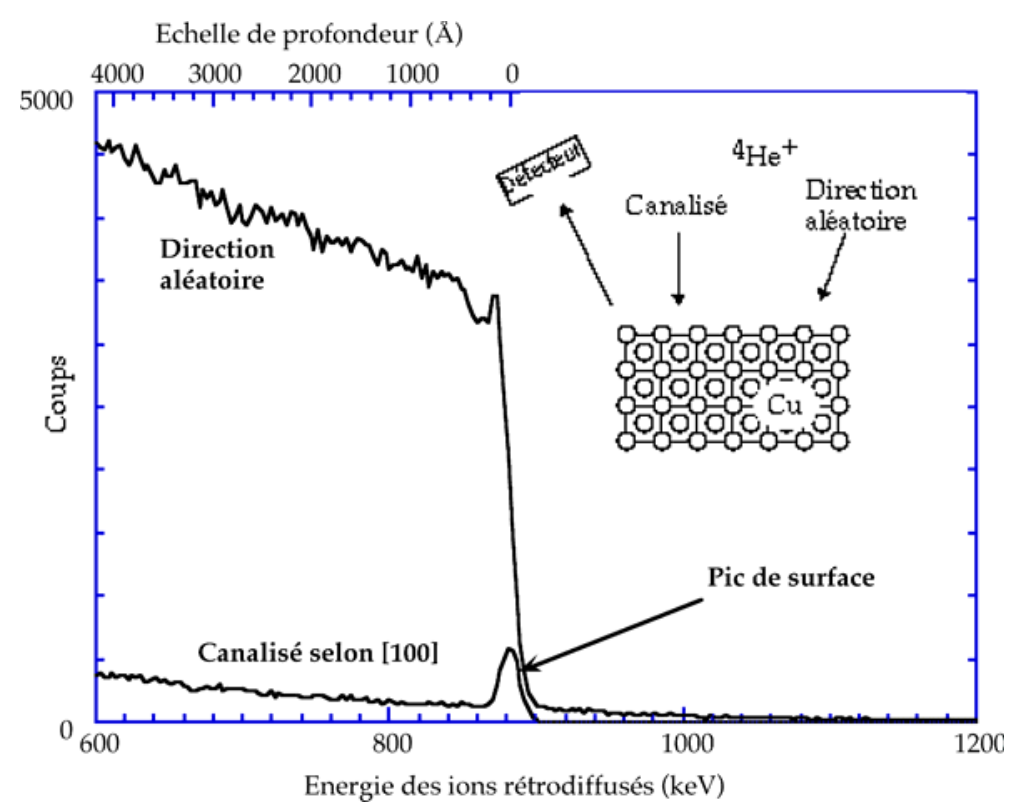

FIG. 1. Spectres RBS hors et en canalisation obtenus sur un monocristal de cuivre avec un faisceau d'ions ${ }^{4} \mathrm{He}$. 


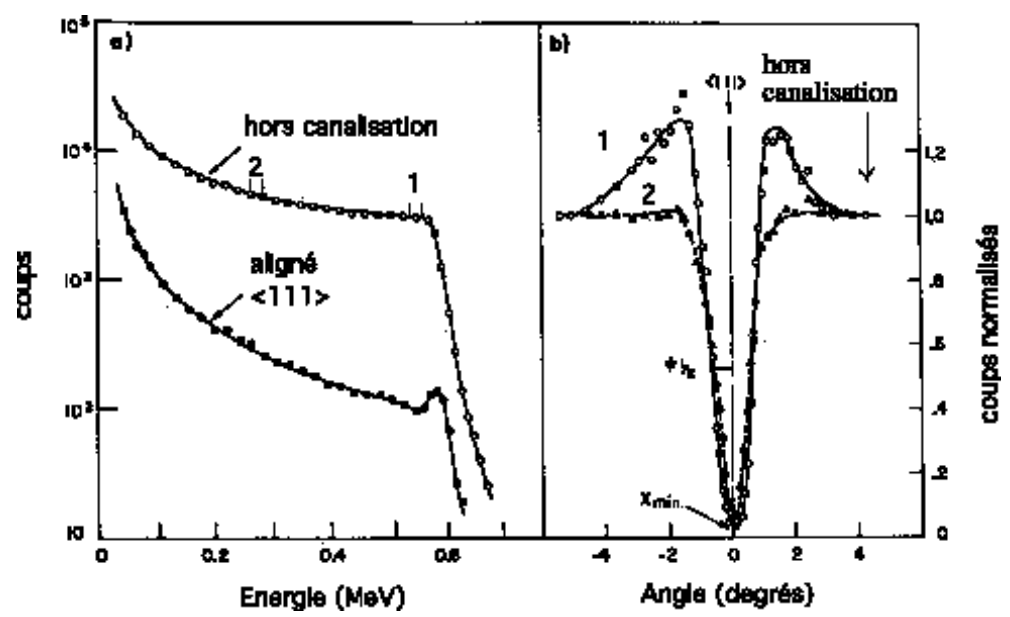

FIG. 2. (a) Spectres RBS hors et en canalisation (échelle logarithmique). (b) Courbes angulaires d'extinction correspondantes : près de la surface (zone 1, Fig. 2a), en profondeur (zone 2, id.).

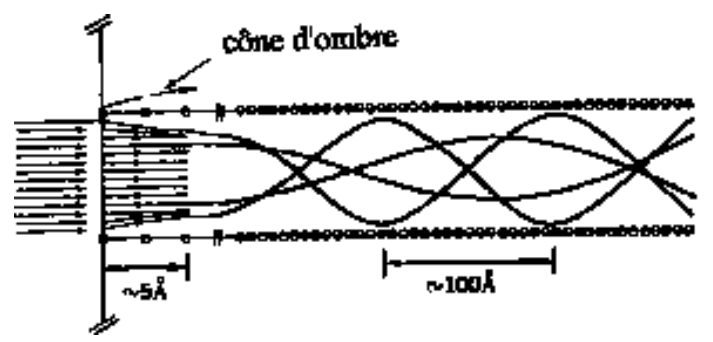

FIG. 3. Représentation schématique des trajectoires des ions canalisés.

\subsection{Grandeurs caractéristiques du phénomène de canalisation}

Une description simple du phénomène consiste à considérer la grandeur «énergie transverse » $E_{\perp}$ d'un ion canalisé. Cette grandeur est définie par la somme de deux termes : l'énergie potentielle d'interaction ion-rangée (ou plan) et l'énergie cinétique associée à la composante perpendiculaire à la rangée ou au plan du vecteur vitesse de l'ion. Pour les ions canalisés, l'angle $\psi$ étant petit, on a $E_{\perp}=U(r)+E \psi^{2} . U(r)$ est estimé à partir du potentiel d'interaction écranté ion-atome, en moyennant sur la rangée considérée :

$$
U(r)=\frac{1}{d} \int_{-\infty}^{+\infty} V\left(\sqrt{z^{2}+r^{2}}\right)
$$

où $d$ est la distance interatomique le long de la rangée. Pour un ion canalisé, en négligeant les pertes d'énergie, $E_{\perp}$ est une constante, déterminée par l'angle $\psi_{o}$ du vecteur vitesse de l'ion et par le paramètre d'impact, par rapport à la rangée ou au plan, à l'entrée dans le cristal. Un ion canalisé a une énergie transverse inférieure à une énergie critique $U\left(r_{o}\right)$ pour laquelle l'ion peut entrer en collision avec un atome de la rangée.

Au fur et à mesure de son parcours, l'ion canalisé subit des interactions et donc des déflexions par les électrons, dont la probabilité de présence dans les canaux n'est pas nulle, et aussi, avec grand paramètre d'impact, par les atomes écartés de leur position moyenne par l'agitation thermique. Ainsi son énergie transverse augmente et l'ion peut ainsi sortir du mode canalisé. C'est le phénomène de décanalisation que l'on observe en comparant un spectre en position de canalisation et un spectre en position hors canalisation. Le rapport $\chi=\frac{Y_{\mathrm{EC}}}{Y_{\mathrm{HC}}}, Y_{\mathrm{EC}}$ et $Y_{\mathrm{HC}}$ étant respectivement les rendements en et hors canalisation, augmente avec la profondeur (Fig. 2a). Une augmentation de l'énergie transverse, et 
donc du $\chi$, peut aussi être due à l'interaction des ions avec des défauts cristallins, qui pourront ainsi être caractérisés.

L'angle critique de canalisation est la demi-largeur caractéristique de la courbe angulaire d'extinction (rendement en fonction de l'angle d'incidence par rapport aux rangées ou aux plans). Il correspond à l'angle d'incidence pour lequel l'énergie transverse est égale à l'énergie critique $U\left(r_{o}\right)$, pour un ion entrant au centre d'un canal. L'expression approximative

$$
\psi_{1}=\sqrt{\frac{2 Z_{1} Z_{2} e^{2}}{E d}}
$$

rend bien compte de la grandeur de ce paramètre. A titre d'exemple, pour des ions ${ }^{4} \mathrm{He}$ de $2 \mathrm{MeV}$ et la direction [100] du silicium, $\psi_{1}$ vaut $0,5^{\circ}$. Un calcul plus précis nécessite la prise en compte de l'amplitude de vibration thermique, laquelle est habituellement prise comme valeur $r_{o}$, et du rayon d'écran d'interaction ion-rangée ou plan.

\subsection{Pic surface}

Les points d'impact des ions incidents arrivant à la surface du cristal sont aléatoirement distribués. Les interactions avec les atomes de la première couche atomique vont avoir le même rendement que si la cible était amorphe. Ce n'est qu'après avoir subi la première déflexion que le phénomène va se produire. Le premier atome d'une rangée, en défléchissant les ions va les empêcher de rencontrer les suivants. C'est l'effet de « cône d'ombre ».

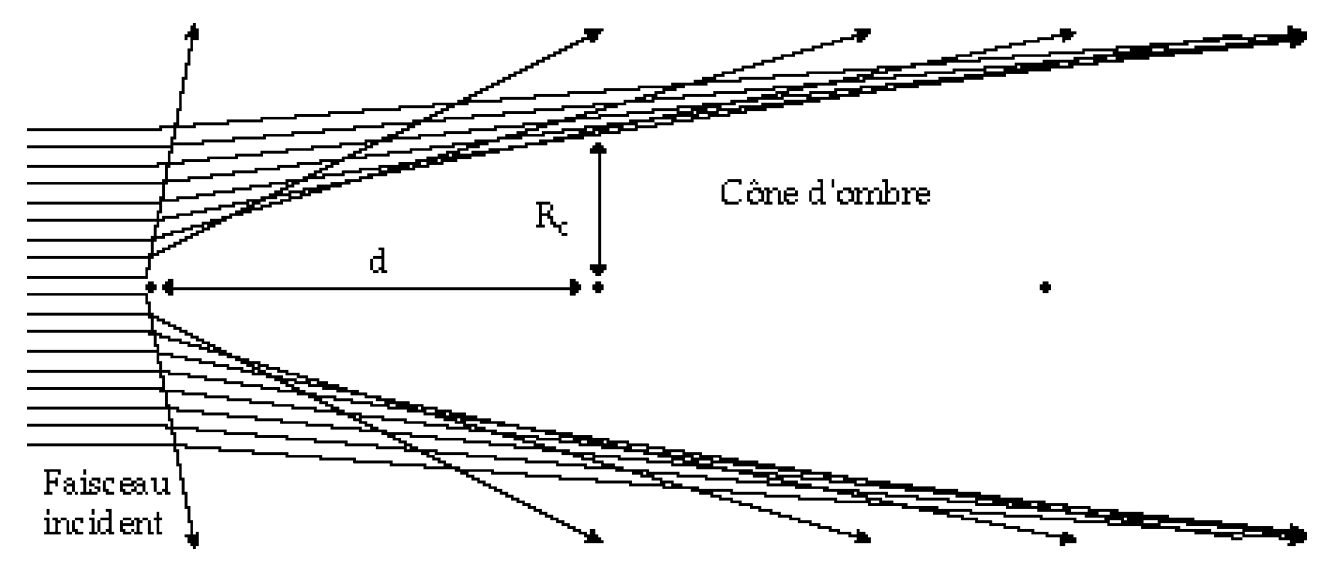

FIG. 4. Représentation schématique de l'effet « cône d'ombre ».

Si les atomes du cristal ne vibraient pas et étaient tous en site, la partie haute énergie du spectre canalisé ne correspondrait qu'aux interactions avec le premier atome de chaque rangée. Ce pic haute énergie du spectre canalisé correspond donc aux interactions avec les atomes de surface. Mais en fait, les atomes du cristal vibrent et même pour le deuxième atome de la rangée, la probabilité d'interaction à petit paramètre d'impact n'est pas nulle et l'intégrale du pic surface représente plus qu'une couche atomique. Cette intégrale va évidemment dépendre du "pouvoir focalisant» qui est représenté par le rayon du cône d'ombre au niveau du deuxième atome de la rangée. On peut calculer ce rayon R à partir de l'approximation de Moliere du potentiel écranté de Thomas-Fermi. Il est très proche de celui calculé à partir du potentiel de Coulomb non écranté $R_{C}=2\left(Z_{1} Z_{2} e^{2} d / E\right)^{1 / 2}$. 


\section{APPLICATIONS À LA CARACTÉRISATION STRUCTURALE DES ÉCHANTILLONS CRISTALLINS}

Au fur et à mesure de la pénétration des ions canalisés, la diffusion sur les électrons et sur les défauts perturbe leur trajectoire, leur énergie transverse augmente, ils sortent du mode canalisé : il y a décanalisation (voir pente du spectre en canalisation). Ainsi, les défauts cristallins vont avoir comme effet d'atténuer le phénomène. La qualité cristalline d'une couche sera caractérisée par la valeur du paramètre $\chi_{\min }$ égal au rapport entre le rendement en canalisation et le rendement hors canalisation. Une étude en fonction de l'angle d'incidence suivant plusieurs directions ou plans cristallins pourra même révéler la dispersion angulaire de position des axes cristallins de la couche (mosaïcité).

Une étude des spectres canalisés va permettre de caractériser les défauts cristallins. Par exemple, une étude en fonction de l'énergie des ions incidents permet d'indiquer si l'on est en présence de défauts ponctuels ou en présence de défauts étendus : une augmentation de la décanalisation avec l'énergie correspond à la présence de défauts étendus (dislocations), l'inverse correspondant à la présence de défauts ponctuels.

\section{1. Étude par canalisation de la localisation en site des impuretés dans les cristaux}

L'étude du phénomène de canalisation selon plusieurs axes cristallographiques va permettre de déterminer la position d'une impureté. Le principe général est simple : si l'impureté est substitutionnelle, le $\chi_{\min }$ sur l'impureté sera identique à celui des atomes du cristal hôte, sinon, une méthode de « triangulation » permettra de déterminer son site.

Une grande précision peut ici être atteinte : la connaissance de la répartition du flux de particules dans les canaux, atteinte par exemple par simulation de Monte Carlo, permet de relier le rendement sur l'impureté à sa position moyenne à quelques $10^{-2} \AA$ près.

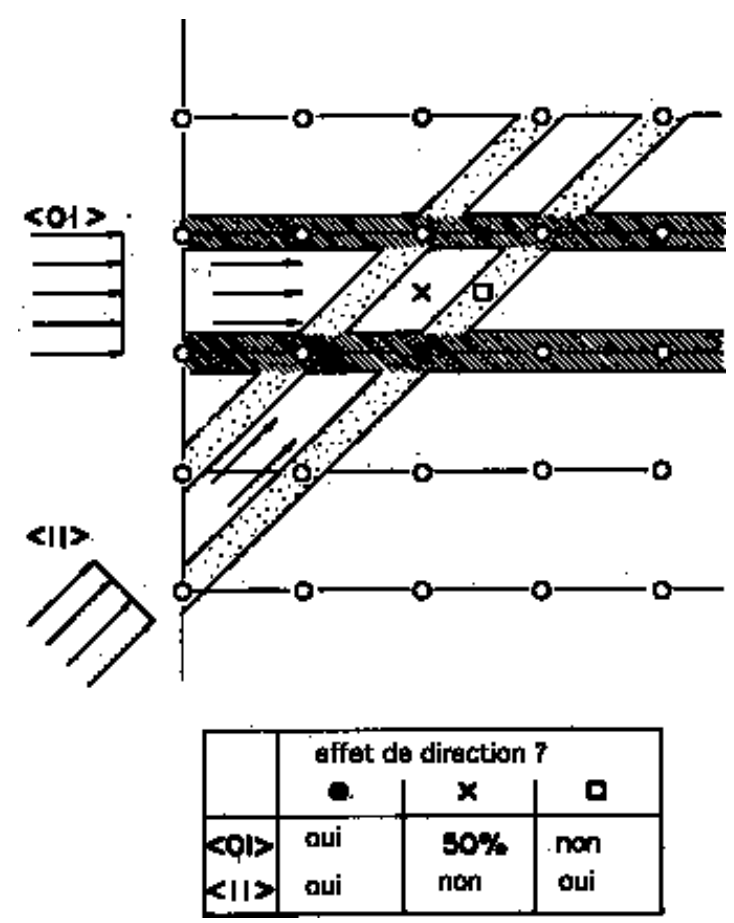

FIG. 5. Schéma à deux dimensions du principe de localisation en site d'une impureté dans un cristal. 


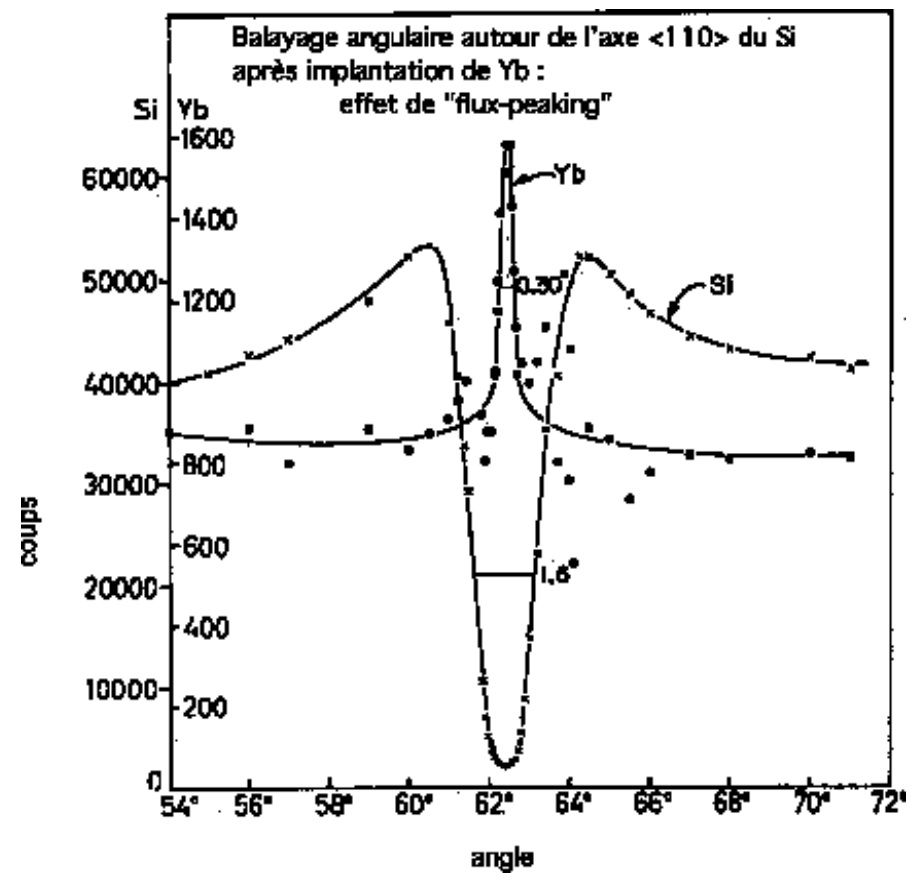

FIG. 6. Localisation de l'ytterbium implanté dans le silicium (après recuit). Les atomes d'Yb sont en site au centre des canaux $<110>$ du silicium. Le pic de rendement sur Yb correspond au fait que le flux de particules est maximum au centre des canaux.

\section{2. Étude par canalisation de l'hétéroépitaxie de couches minces}

La croissance d'une couche cristalline sur un cristal s'accompagne d'une contrainte de celle-ci, les paramètres cristallins de l'une et de l'autre n'étant pas identiques. Pour des couches suffisamment minces les contraintes ne sont pas relâchées par des défauts étendus. Très souvent, on est en présence d'un axe de la couche aligné avec un axe de du cristal sous-jacent, les autres axes étant décalés angulairement. En repérant par des balayages angulaires les positions angulaires des uns et des autres, on peut déterminer ces décalages et ainsi mesurer, via les paramètres élastiques, les contraintes subies par la couche.
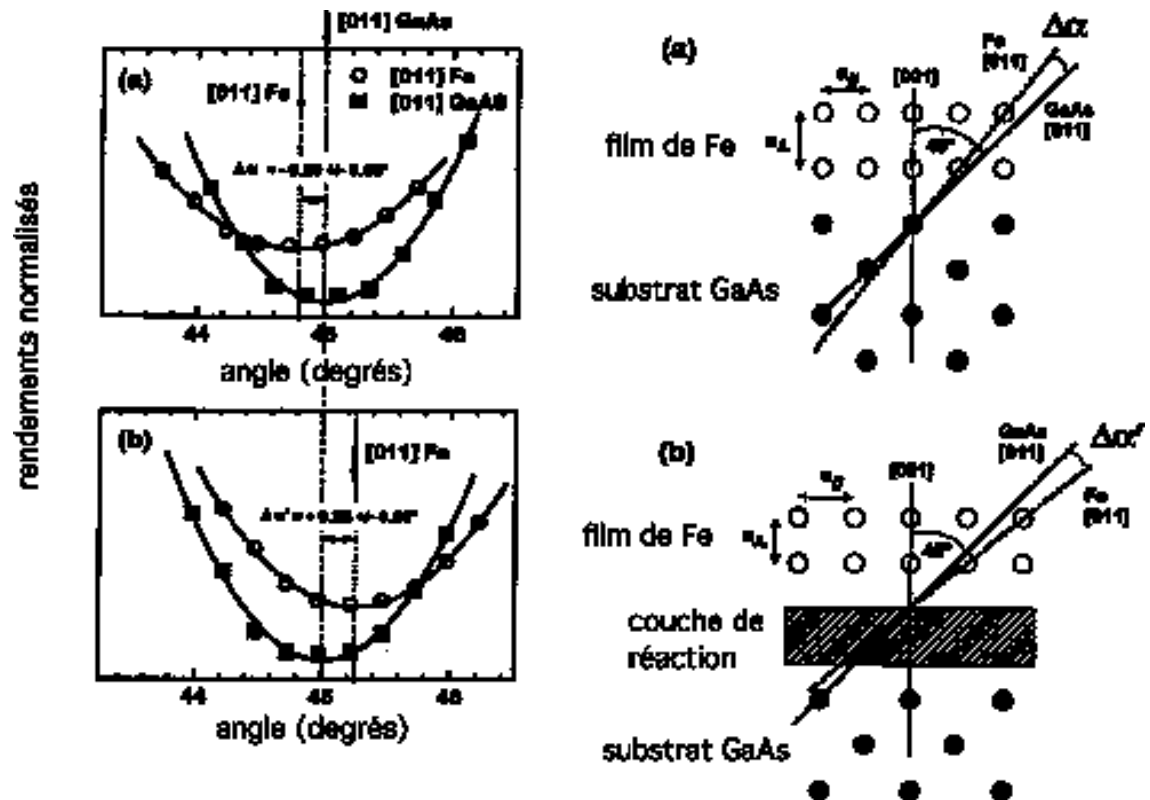

FIG. 7. Étude de l'épitaxie d'une couche de fer sur AsGa (a) et sur une couche où Fe et GaAs ont réagi (b). Les mesures indiquent que le film de fer est contraint, parallèlement à la surface, en compression dans le cas (a) et en extension dans le cas (b). 


\section{3. Étude par canalisation des surfaces et interfaces}

\subsubsection{Surfaces}

L'étude du (ou des) pic surface va permettre de caractériser la qualité cristalline des toutes premières couches atomiques d'un cristal. Par calcul, notamment par simulation de Monte-Carlo, on connaît le nombre d'atomes par rangée qui détermine l'intégrale du pic surface pour le cristal parfait. Toute augmentation par rapport à ce nombre est représentative d'un désordre des premières couches cristallines et permet de quantifier ce désordre. Ce type d'étude peut être particulièrement utile, par exemple, lors de la recherche de traitements de surface visant à obtenir un très bon ordre cristallin de surface en vue, par exemple, d'épitaxie ou d'hétéroépitaxie.

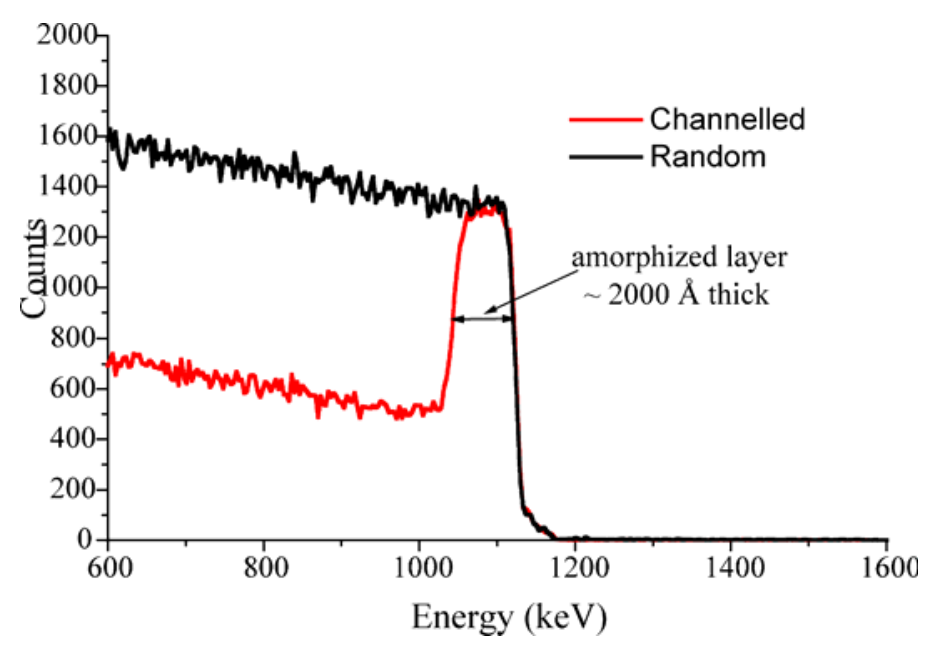

FIG. 8. Exemple de détermination de l'épaisseur amorphisée par implantation ionique dans un cristal de silicium.

\subsubsection{Interfaces}

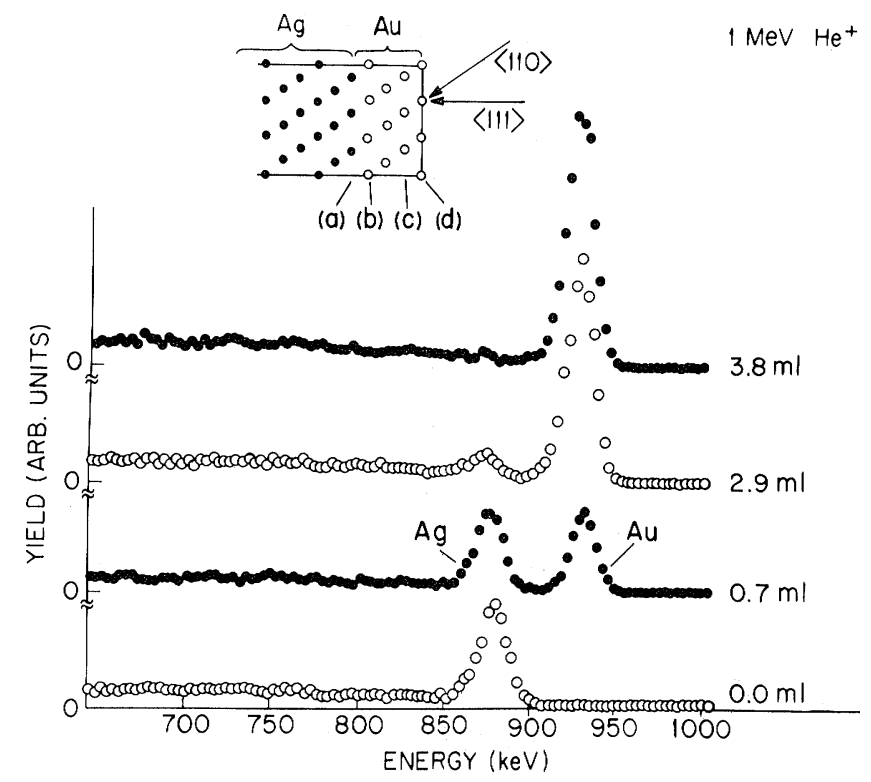

FIG. 9. Spectres en canalisation par rapport au substrat Ag pour des dépôts successifs de monocouches atomiques d'or. Pour 0,7 et 2,9 monocouches d'or déposées sur le cristal d'argent, on distingue les deux pics surface. 
Dans le cas d'épitaxie ou d'hétéroépitaxie, l'étude des spectres en canalisation permet de mettre en évidence les défauts d'interface. Dans le cas de couches minces bien cristallisées sur un substrat cristallin, on peut étudier non seulement le pic surface de la couche, la décanalisation dans la couche, représentative des défauts et de leur répartition en profondeur, mais aussi le pic « surface » du substrat. Ce dernier est représentatif, à la fois du désordre d'interface du côté substrat et du désalignement couche-substrat. Un exemple particulièrement intéressant est le cas d'un alignement parfait des rangées cristallines de la couche et du substrat. C'est ce que l'on observe lors du dépôt d'or sur argent : le pic «surface » Ag disparaît dès que quelques monocouches d'Au ont été épitaxiées, à cause de l'effet d'ombre des atomes d'or (Fig. 9 ).

\section{UTILISATION DE LA CANALISATION POUR L'ÉTUDE DES COUCHES MINCES AMORPHES SUR UN SUBSTRAT CRISTALLIN}

Une application simple mais non structurale de la canalisation est la révélation, par augmentation du rapport signal sur bruit, de la composition d'une couche mince amorphe sur un substrat cristallin. En effet, si on aligne le faisceau avec un axe cristallographique de bas indices du substrat, la contribution de celui-ci au spectre va pouvoir être réduite d'au moins un ordre de grandeur, laissant le signal correspondant aux éléments de la couche. On peut ainsi, dans certains cas difficiles, arriver à augmenter beaucoup la précision des mesures.

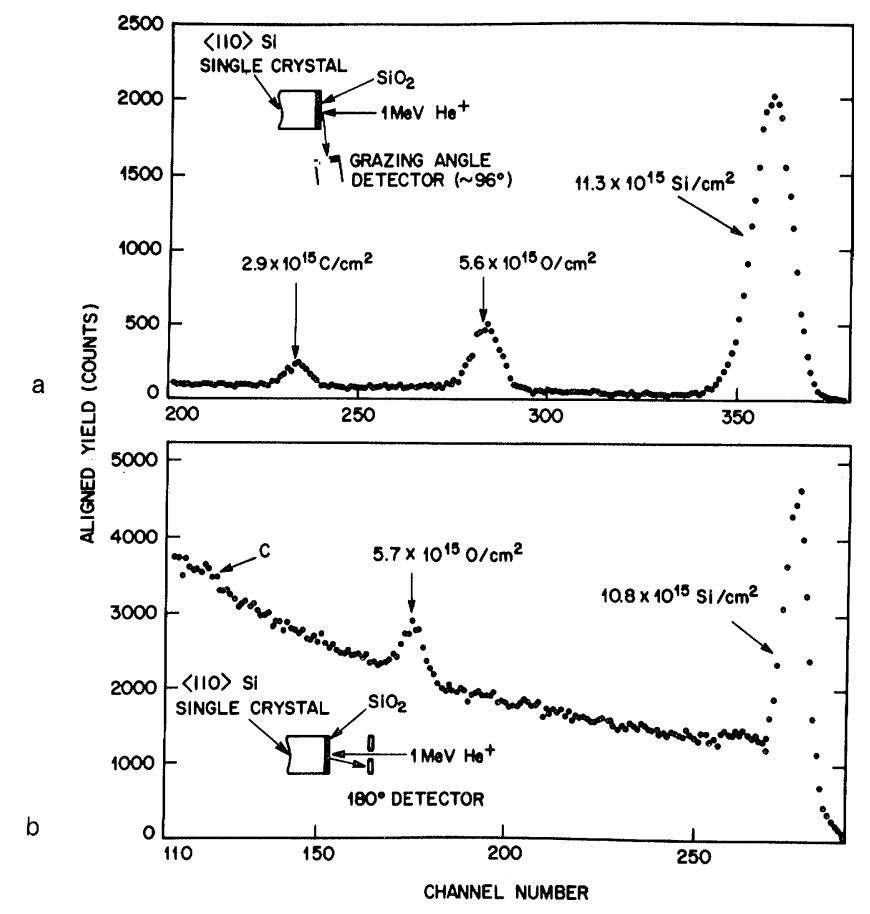

FIG. 10. Exemple d'utilisation du phénomène de canalisation pour réduire le signal RBS du substrat monocristallin. Ici, pour $\mathrm{SiO}_{2} / \mathrm{Si}$, il est montré l'intérêt de se placer de plus en détection rasante. 


\section{BIBLIOGRAPHIE (QUELQUES OUVRAGES AVEC BEAUCOUP DE RÉFÉRENCES À L'INTÉRIEUR)}

L.C. Feldman et J.W. Mayer, Fundamentals of surface and thin films analysis, North-Holland (1986). W.K. Chu, J.W. Mayer et M.A. Nicolet, Backscattering spectrometry, Academic Press, New York (1978).

L.C. Feldman, J.W. Mayer et S.T. Picraux, Materials analysis by ion channeling, Academic Press, New York (1982).

J.R. Tesmer et M. Nastasi Eds., Handbook of modern ion beam analysis, Material Research Society (1995).

J.W. Mayer et E. Rimini, Ion beam handbook for material analysis, Academic Press, New York (1977).

B. Agius, M. Froment et co-auteurs, Surfaces, interfaces et films minces. Observation et analyse, Dunod (1990).

J.M. Walls Ed., Methods of surface analysis, Cambridge University Press (1989).

J. Tirira, Y. Serruys et P. Trocellier, Forward recoil spectroscopy. Applications to hydrogen determination in solids, Plenum Press (1996). 